\title{
Management of Pediatric Traumatic Macular Holes - Case Report
}

\author{
S. Azevedo N. Ferreira A. Meireles \\ Department of Ophthalmology, Santo António Hospital, Oporto, Portugal
}

\section{Key Words}

Macular hole $\cdot$ Trauma $\cdot$ Pediatric patients

\begin{abstract}
Purpose: To report and describe the clinical course of four pediatric traumatic macular hole $(\mathrm{TMH})$ cases and respective management. Case Report: Four pediatric patients with macular hole following blunt ocular trauma underwent early pars plana vitrectomy, with consecutive hole closure. Initial visual acuity was $<20 / 400$ or worse in all patients. After surgery, all patients had visual acuity improvement, with final visual acuity being $>20 / 50$ in 2 of the patients by the end of the follow-up. Macular hole closure was achieved in all patients with a single procedure, and anatomical success was confirmed by optical coherence tomography until the end of the follow-up. Conclusions: Although spontaneous closure of TMH is not uncommon, especially in pediatric patients, early pars plana vitrectomy seems to be a safe and effective choice in pediatric TMH management. The risk/benefit ratio of surgery seems to be better than observation.
\end{abstract}

\section{Introduction}

The pathogenesis of traumatic macular hole is not fully understood and the best treatment guidelines are controversial, especially in pediatric patients. Although most macular holes are age-related idiopathic conditions, that affect mainly female patients, they may also occur in association with trauma, thus being called traumatic macular holes (TMH). As opposed to idiopathic macular holes, TMH are not associated with gradual onset and the mechanism of their formation remains controversial. Some authors theorized that the force of the impact transmitted to the macula could result in rupture of the fovea [1]. Another hypothesis is that sudden vitreous separation could be the cause of TMH, although most cases actually have an attached posterior vitreous [1-3]. Other factors that may be involved are: outward expansion of the equator, flattening and subsequent posterior displacement of 
the posterior pole, trampoline-like movement, tangential tractional forces on retinal surface, contusion necrosis, cystoid degeneration, subfoveal hemorrhage, choroidal rupture, and anterioposterior vitreous traction. Optical coherence tomography (OCT) allowed the detection of subclinical inner retinal layers changes that could play a role in the pathogenesis of TMH formation [4].

Besides the mechanism of TMH formation, the decision of whether to operate or simply observe these TMH is also controversial. Several retrospective case series have shown positive outcomes of pars plana vitrectomy for TMH [3, 5-11]. On the other hand, many cases of spontaneous closure of TMH have also been reported in the literature [12-19].

Spontaneous closure can more easily occur in children, in small macular holes (100-200 $\mu \mathrm{m})$, if the posterior hyaloid remains attached, or if no fluid cuff is present. Although unpredictable, it usually occurs between 3 and 6 months, and is related with final visual acuity (fVA) $\geq 20 / 40$ in $50 \%$ of the patients [14-20]. The mechanism of spontaneous TMH closure encompasses the proliferation of glial cells or retinal pigment epithelial (RPE) cells from each bank of the hole's edge to fill the hole bottom, and stimulation of astrocyte migration to heal the TMH.

We report four cases of pediatric TMH that underwent surgical repair consisting of early standard 3-port pars plana vitrectomy with posterior hyaloid removal, internal limiting membrane peeling and gas tamponade. All patients were advised to maintain prone position for at least six hours a day for five days.

Our interest in reporting this case series is to highlight the effectiveness and benefits of an early interventional approach, instead of an expectant/observational one.

\section{Case 1}

A 12-year-old boy presented with sudden unilateral visual loss following a blunt trauma with a bottle cork in a mildly amblyopic left eye. At presentation, the initial visual acuity (iVA) was hand motion in the left eye and 20/20 in the right eye. Anterior segment examination revealed hyphema, corneal edema and red fundus reflex absence. The patient underwent an ocular ultrasound which excluded the presence of a retinal detachment. Eight days later the child was reassessed: no hyphema was identified, visual acuity (VA) remained hand motion and a macular hole was detected on fundus biomicroscopy (fig. 1). An OCT was performed which confirmed a full-thickness macular hole (fig. 2) with posterior vitreous attachment. One day later the patient underwent surgery as described above. One month after surgery, the OCT confirmed closure of the macular hole and VA had improved to 20/50, which remained stable until the end of the follow-up.

\section{Case 2}

A 10-year-old girl was hit by a soccer ball followed by sudden visual loss in the left eye. At that time the VA was 20/400 in the left eye and 20/20 in the right eye. Anterior segment examination was unremarkable. Fundus biomicroscopy revealed a lamellar hole, confirmed by OCT (fig. 3), which turned into a full-thickness macular hole identified 8 days after trauma (fig. 4). Surgery was performed the following day. One week after surgery, fundus examination revealed closure of the macular hole, and by the end of the first month, the best corrected VA (BCVA) was 20/50. One year later the VA was 20/30, with maintenance of macular hole closure (fig. 5). 


\section{Case 3}

A 9-year-old boy was hit by a soccer ball with sudden visual loss in the left eye. iVA was $20 / 400$ in the left eye and 20/20 in the right eye. Anterior segment examination showed no lesions. Fundus biomicroscopy revealed a macular hole, confirmed by OCT (fig. 6). Surgery was performed the following day. One week later, OCT confirmed closure of the macular hole. One month later, the BCVA was 20/50. After 6 months of follow-up, the VA was 20/40, with maintenance of macular hole closure (fig. 7).

\section{Case 4}

A 15-year-old boy was hit on his left eye by a soccer ball. At presentation, iVA of the affected eye was hand motion. The adnexa and anterior segment were unremarkable. Fundoscopy showed little intraretinal hemorrhages, commotio retinae in the posterior pole, and the presence of a macular hole. The OCT revealed a full-thickness macular hole with hyaloid still attached (fig. 8). The patient underwent surgery 2 days later. Five days after surgery, the OCT confirmed macular hole closure (fig. 9) and the VA was counting fingers. Follow-up observations showed sustained heterogeneous areas of retinal pallor mingled with hyperpigmentation zones and maintenance of macular hole closure. The OCT revealed disorganization of the retinal layers with foveal atrophy and disruption of the photoreceptor layer. The VA remained in counting fingers until the end of the follow-up.

\section{Discussion}

Very little can be found in the literature regarding pediatric TMH management and its results compared to adult population.

Wu et al. [21] presented a case series with TMH in which 13 pediatric patients (1-15 years old) were submitted to plasmin-assisted vitrectomy, membrane peeling and gas or silicone oil injection, followed by prone position for 7 days. The macular hole closed in 12 (92\%) patients. fVA could be measured only in 12 of 13 patients. From those, 11 (92\%) patients had VA improvement of 2 or more lines and six (50\%) achieved vision of 20/50 or better; all patients achieved a vision better than 20/200.

Wachtlin and associates [22] reported a case series of four pediatric patients with TMH (10-15 years old). All patients underwent pars plana vitrectomy with platelet concentrate, internal limiting membrane (ILM) peeling, and gas tamponade for stage $3 \mathrm{TMH}$ repair. Primary closure was achieved by a single intervention in all patients, with a visual improvement of three to seven lines after surgery. The surgically achieved visual improvement remained stable during the follow-up and no complications were reported.

In 2007, Brasil and Brasil [1] reported a single case of a 9-year-old boy with a TMH who gained vision from $20 / 300$ to $20 / 70$ after surgery with ILM peeling and silicone oil tamponade, which remained stable one year after the silicone oil removal.

On the other hand, the surgical outcomes of TMH in adults have been analyzed in multiple retrospective case series. Closure rate varies from 92 to $100 \%$ and fVA $>20 / 40$ from 38 to $48 \%$ [5-10].

Rubin et al. [5] presented a case series of 12 consecutive patients with TMH who underwent vitrectomy, fluid-gas exchange and instillation of bovine or recombinant transforming growth factor beta- 2 . The closure rate was $92 \%$, repeated surgery having been necessary 
in 3 patients. The BCVA improved by at least 2 lines in 8 (67\%) of 12 eyes. Six (50\%) of 12 eyes improved to $20 / 40$ or better.

Another reference of the use of platelet concentrate was reported by García-Arumí and associates [7] who presented the surgical outcomes of 14 eyes with full-thickness TMH submitted to pars plana vitrectomy with posterior hyaloid dissection, followed by complete fluid-gas exchange, and instillation of $0.1 \mathrm{ml}$ of platelet concentrate just over the macular hole. Closure rate was 93\% (13 of 14 eyes), with a vision improvement of at least 4 Snellen lines in every closed hole. The mean fVA was 20/30, and two patients had a fVA of 20/20. No intra- or postoperative complications were noted.

Several studies have demonstrated the benefits of vitrectomy without adjunctive therapy, but almost exclusively in adults.

In the series of Amari et al. [10] and Chow and associates [9] the final closure rate of 96 and $94 \%$, respectively, and a VA improvement of at least two lines in more than $70 \%$ of patients were obtained without any adjunctive therapy.

In 2001, Kuhn et al. [11] reported the benefits of ILM removal in a case series of 17 consecutive TMH patients. All patients were submitted to vitrectomy and ILM removal with no adjuvant therapy. Results showed $100 \%$ closure rate. Vision improved by at least 2 Snellen lines in 16 (94\%) eyes.

More recently, a multicenter case series of $25 \mathrm{TMH}$ cases was published, and the authors observed a $96 \%$ closure rate and a vision of $20 / 50$ or better in $64 \%$ of the eyes [1].

Some cases of TMH spontaneous resolution have also been published. In total we found 37 cases of spontaneous TMH closure and the majority of them occurred in children [12-18, 23-26]. All the holes closed within up to one year, and 55\% within up to three months of trauma [12-18, 23-26]. Therefore, some authors advocate at least an initial period of observation instead of an early intervention [1]. Although our series is too small to postulate the benefits of an early intervention some features should be highlighted. The iVA in the eyes with spontaneously closed TMH was $20 / 400$ or better in $70.3 \%$, the size of the hole was equal to or less than 0.2 disk diameter (DD) in at least $75.7 \%$ of the eyes and $40.5 \%$ of the patients were more than 15 years old. On the contrary, all our patients had iVA of 20/400 or worse, the macular hole size was superior to $0.3 \mathrm{DD}$ and our oldest patient was 15 years old. Therefore, spontaneous closures of TMH described are mostly in patients with small holes and VA above 20/400. Larger holes seem to result from higher kinetic energy which causes more severe retinal edema, RPE damage, subretinal bleeding with its possible photoreceptor toxicity, and retinal necrosis with tissue loss. These factors may contribute to inability to reappose the retinal edges by the contracting fibroglial tissue, limiting the reparative process to flattening of the retinal edges surrounding the hole [17]. From the 37 cases described, only 11 had fluid cuff described, and all but one had the posterior hyaloid attached, thus having a higher probability to spontaneously close. Also the patients that ended up with a BCVA $\geq 20 / 25$ (12 patients) had holes $\leq 0.2$ DD, initial BCVA between $20 / 200$ and $20 / 40$, and only two had fluid cuff present.

Regarding only the case series that reported a spontaneous closure rate of TMH $[15,24$, 27] one can say that most of them did not close spontaneously: only 13 of 34 (38.2\%) TMH resolved spontaneously. Few data is found regarding the numbers of non-closing TMH managed with an observational attitude. What size were they? What were their anatomical features? How old were the patients? What was the initial BCVA? And most of all, what was the final BCVA on holes that remained unclosed? Would these patients benefit in the same way with surgery after waiting so long?

TMH are less frequent than idiopathic ones, have a different pathogenesis and do not have a well-established management guideline, especially in pediatric patients. However, 
several well-documented retrospective case series have actually shown very similar surgical outcomes for idiopathic and TMH [20, 24, 27]. The use of platelet concentrate and autologous plasmin has been described, although more studies are necessary.

Some authors claim that an observation period of 3 to 6 months is advised, especially in young patients with small holes, good iVA and posterior vitreous adhesion to the TMH edges due to the higher possibility of spontaneous resolution. In the remaining cases the possibility of spontaneous closure is low [14-20].

The photoreceptor damage is more likely, the longer the delay. The consequences of a long period of waiting for the spontaneous closure of the macular hole have not been clarified. Few series have been published concerning this matter, all of them with few cases and controversial results. Many questions remain controversial and unanswered. Until when should we wait? Authors have reported the enlargement of macular hole during the followup [14] with unclear consequences. Which is the best timing to intervene? Which TMH are more suitable to spontaneously close? Is the probability the same regardless of the child's age? Should we wait in patients in amblyopic ages, in patients with only one eye, or in patients with previous low vision? What impact could this situation have in the psychomotor development in these ages?

In conclusion, early pars plana vitrectomy with ILM peeling and gas tamponade seems to be a safe and effective choice for the therapy of TMH in pediatric patients. When compared to spontaneous closure results found in the literature, the surgical closure rate is higher and the fVA is slightly better after vitrectomy. We believe that early vitrectomy could facilitate and accelerate the closure process, preventing irreversible structural changes and consequent permanent damage.

High-resolution cross-sectional retinal images provided by OCT add new data to the understanding of pathogenesis of TMH and may help in management decisions.

\section{References}

1 Brasil OFM, Brasil OM: Management of traumatic macular holes: case report. Arq Bras Oftalmol 2008;71:581-584.

2 Delori F, Pomerantzeff 0, Cox MS: Deformation of the globe under high-speed impact: its relation to contusion injuries. Invest Ophthalmol 1969:8:290-301.

-3 Johnson RN, McDonald HR, Lewis H, Grand MG, Murray TG, Mieler WF, et al: Traumatic macular hole: observations, pathogenesis, and results of vitrectomy surgery. Ophthalmology 2001;108:853-857.

-4 Oehrens AM, Stalmans P: Optical coherence tomographic documentation of the formation of a traumatic macular hole. Am J Ophthalmol 2006;142:866-869.

5 Rubin JS, Glaser BM, Thompson JT, Sjaarda RN, Pappas SS, Murphy RP: Vitrectomy, fluid-gas exchange and transforming growth factor-beta-2 for the treatment of traumatic macular holes. Ophthalmology $1995 ; 102: 1840-1845$.

7 De Bustros S: Vitreous surgery for traumatic macular hole. Retina 1996;16:451-452.

7 García-Arumí J, Corscostegui B, Cavero L, Sararols L: The role of vitreoretinal surgery on the treatment of posttraumatic macular hole. Retina 1997;17:372-377.

-8 Margherio AR, Margherio RR, Hartzer M, Trese MT, Williams GA, Ferrone PJ: Plasmin enzyme-assisted vitrectomy in traumatic pediatric macular holes. Ophthalmology 1998;105:1617-1620.

$\checkmark 9$ Chow DR, Williams GA, Trese MT, Margherio RR, Ruby AJ, Ferrone PJ: Successful closure of traumatic macular holes. Retina 1999;19:405-409.

10 Amari F, Ogino N, Matsumura M, Negi A, Yoshimura N: Vitreous surgery for traumatic macular holes. Retina 1999;19:410-413.

11 Kuhn F, Morris R, Mester V, Witherspoon CD: Internal limiting membrane removal for traumatic macular holes. Ophthalmic Surg Lasers 2001;32:308-315.

-12 Kusaka S, Fujikado T, Ikeda T, Tano Y: Spontaneous disappearance of traumatic macular holes in young patients. Am J Ophthalmol 1997;123:837-839.

-13 Parmar DN, Stanga PE, Reck AC, Vingerling JR, Sullivan P: Imaging of a traumatic macular hole with spontaneous closure. Retina 1999;19:470-472. 


\begin{tabular}{l|l}
\hline Case Rep Ophthalmol 2013;4:20-27 \\
\hline DOI: $10.1159 / 000350543$ & $\begin{array}{l}\text { ○ } 2013 \text { S. Karger AG, Basel } \\
\text { www.karger.com/cop }\end{array}$ \\
\hline
\end{tabular}

Azevedo et al.: Management of Pediatric Traumatic Macular Holes - Case Report

14 Mitamura Y, Saito W, Ishida M, Yamamoto S, Takeuchi S: Spontaneous closure of traumatic macular hole. Retina 2001;21:385-389.

15 Yamashita T, Uemara A, Uchino E, Doi N, Ohba N: Spontaneous closure of traumatic macular hole. Am J Ophthalmol 2002;133:230-235.

16 Yamada H, Sakai A, Yamada E, Nishimura T, Matsumura M: Spontaneous closure of traumatic macular hole. Am J Ophthalmol 2002;134:340-347.

17 Yeshurun I, Guerrero-Naranjo JL, Quiroz-Mercado H: Spontaneous closure of a large traumatic macular hole in a young patient. Am J Ophthalmol 2002;134:602-603.

18 Menchini U, Virgili G, Giacomelli G, Cappelli S, Giansanti F: Mechanism of spontaneous closure of traumatic macular hole: OCT study of one case. Retina 2003;23:104-106.

19 Carpineto P, Ciancaglini M, Aharrh-Gnama A, Agnifili L, Cerulli AM, Cirone D, et al: Optical coherence tomography and fundus microperimetry imaging of spontaneous closure of traumatic macular hole: a case report. Eur J Ophthalmol 2005;15:165-169.

20 Smiddy WE: The current status of macular hole surgery. Bull Soc Belge Ophthalmol 1996;262:31-42.

-21 Wu WC, Drenser KA, Trese MT, Williams GA, Capone A: Pediatric traumatic macular hole. Results of autologous plasmin enzyme-assisted vitrectomy. Am J Ophthalmol 2007;144:668-672.

-22 Wachtlin J, Jandeck C, Potthöfer S, Kellner U, Foerster MH: Long-term results following pars plana vitrectomy with platelet concentrate in pediatric patients with traumatic macular hole. Am J Ophthalmol 2003;136:197-199.

23 Bosch-Valero J, Mateo J, Lavilla-García L, Núñez-Benito E, Cristóbal JA: Spontaneous closure of full thickness traumatic macular holes. Arch Soc Esp Oftalmol 2008;83:325-327.

24 Tomii A, Ikeda N, Kurusu A, Mimura O: Clinical course of traumatic macular hole. Jpn Rev Clin Ophthalmol 1999;53:1274-1278.

25 Carpineto P, Ciancaglini M, Aharrh-Gnama A, Agnifili L, Cerulli AM, Cirone D, Mastropasqua L: Optical coherence tomography and fundus microperimetry imaging of spontaneous closure of traumatic macular hole: a case report. Eur J Ophthalmol 2005;15:165-169.

26 Nasr MB, Symeonidis C, Tsinopoulos I, Androudi S, Rotsos T, Dimitrakos SA: Spontaneous traumatic macular hole closure in a 50-year-old woman: a case report. J Med Case Reports 2011;5:290.

27 Mizusawa Y, Ichibe M, Yoshizawa T, Ando N: Clinical evaluation of traumatic macular hole. Jpn Rev Clin Ophthalmol 1996;90:790-792.
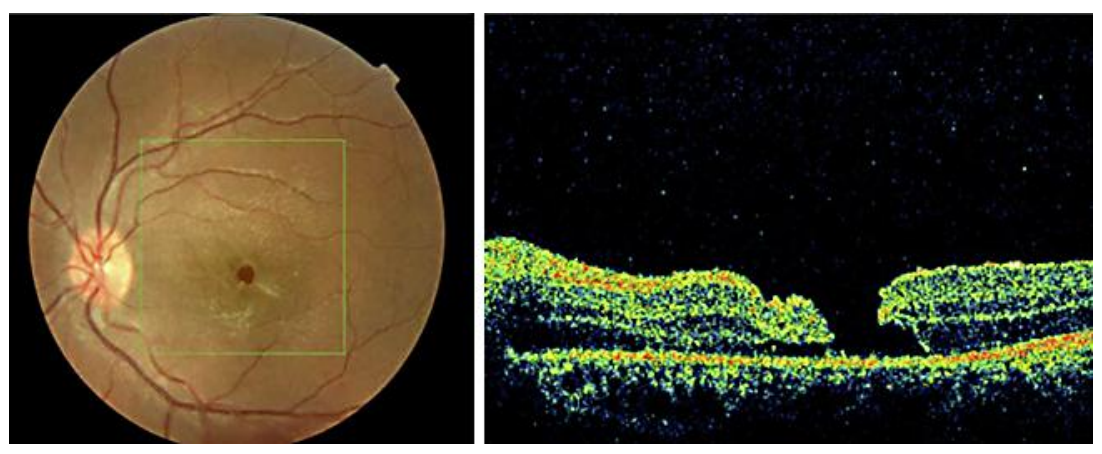

Fig. 1. Presentation: OS, full-thickness macular hole, iVA hand motion. 
Case Reports in

Ophthalmology

\begin{tabular}{l|l}
\hline Case Rep Ophthalmol 2013;4:20-27 \\
\hline DOI: 10.1159/000350543 & $\begin{array}{l}\text { @ } 2013 \text { S. Karger AG, Basel } \\
\text { www.karger.com/cop }\end{array}$ \\
\hline
\end{tabular}

Azevedo et al.: Management of Pediatric Traumatic Macular Holes - Case Report
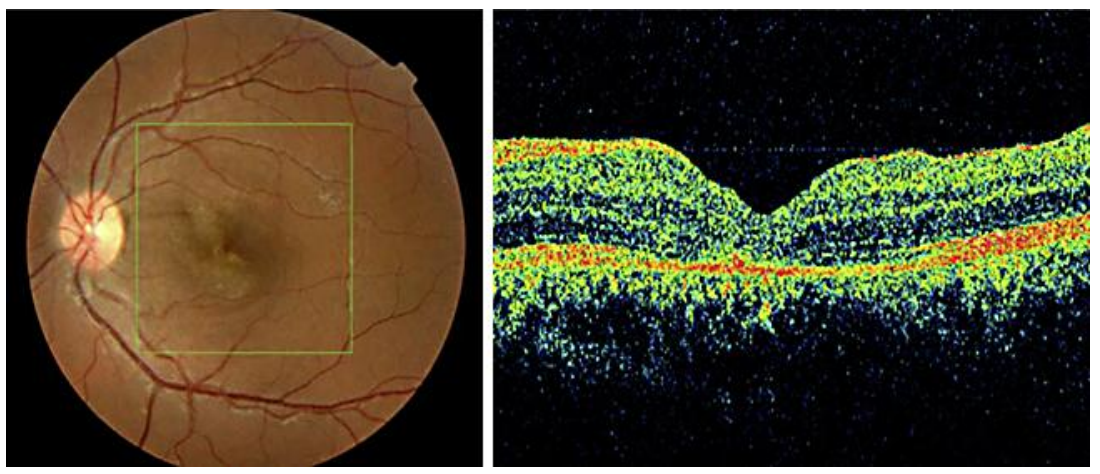

Fig. 2. One year later: macular hole closure, fVA 20/50.
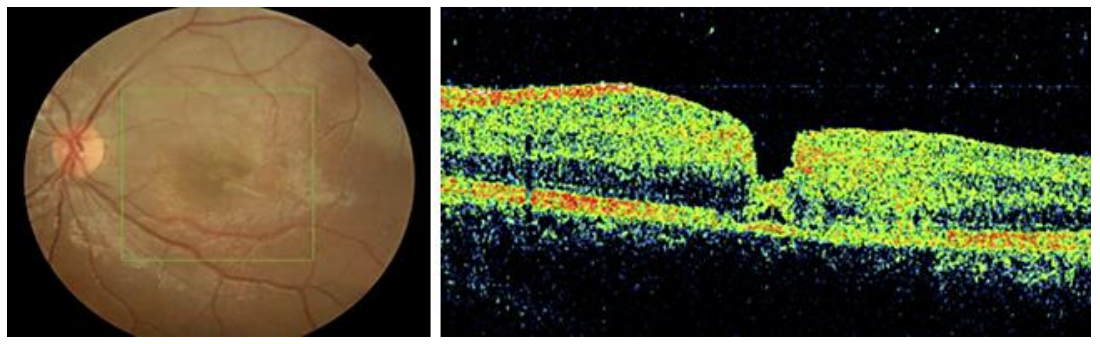

Fig. 3. Presentation: OS, lamellar hole, iVA 20/200.
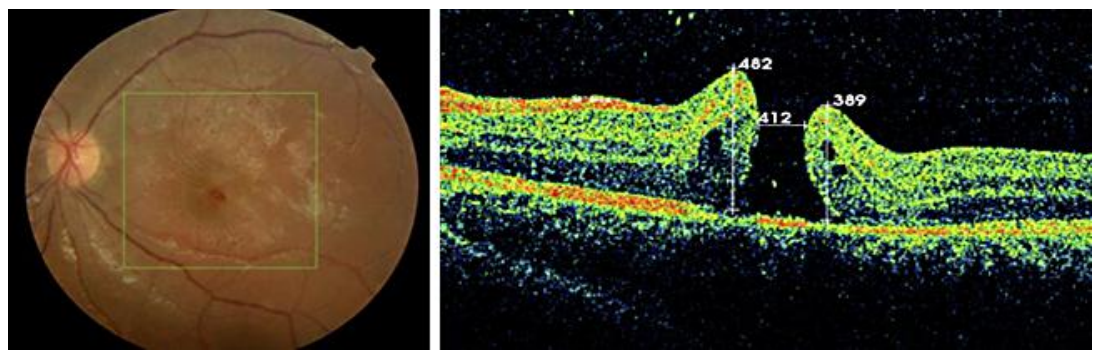

Fig. 4. Eight days after trauma: OS, full-thickness macular hole, VA 20/200.
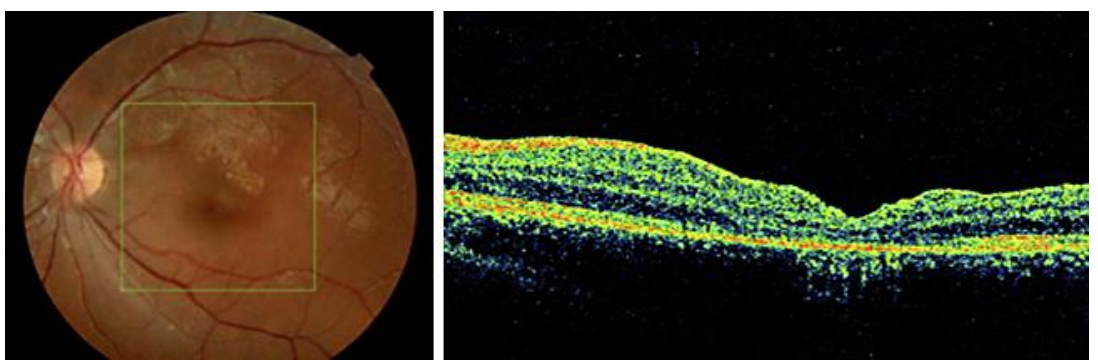

Fig. 5. One year later: macular hole closure, fVA 20/30. 
Case Reports in

Ophthalmology
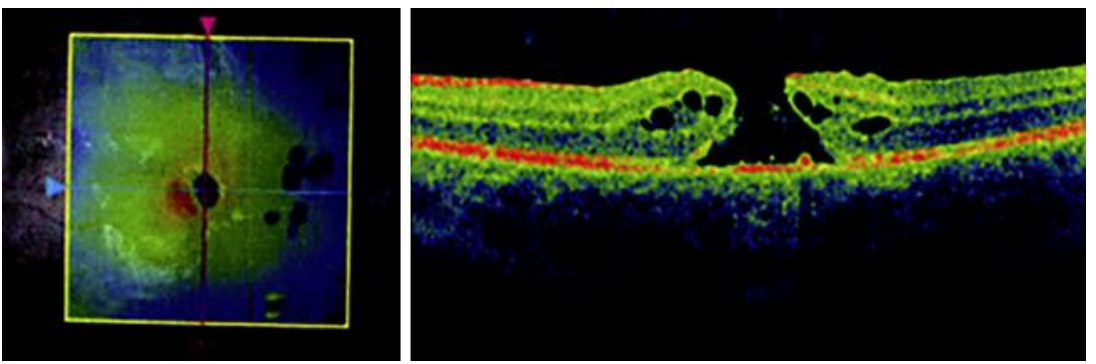

Fig. 6. Presentation: full-thickness macular closure iVA: 20/200.
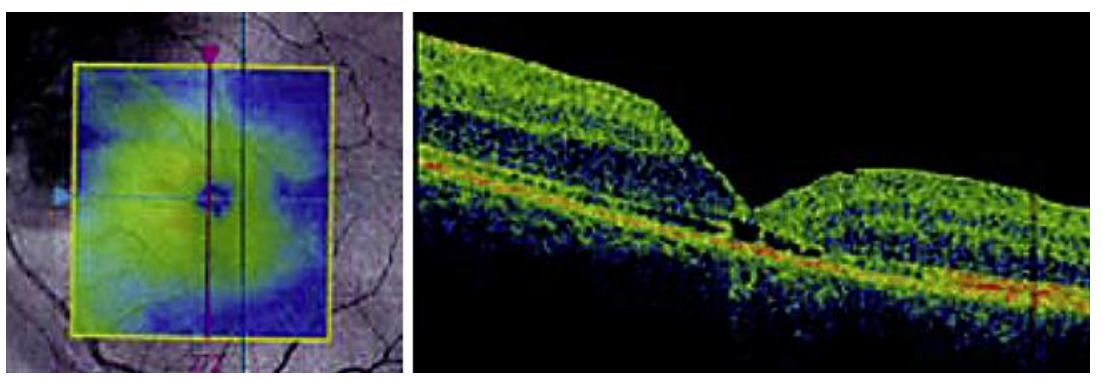

Fig. 7. Six months later: macular closure fVA: 20/40.
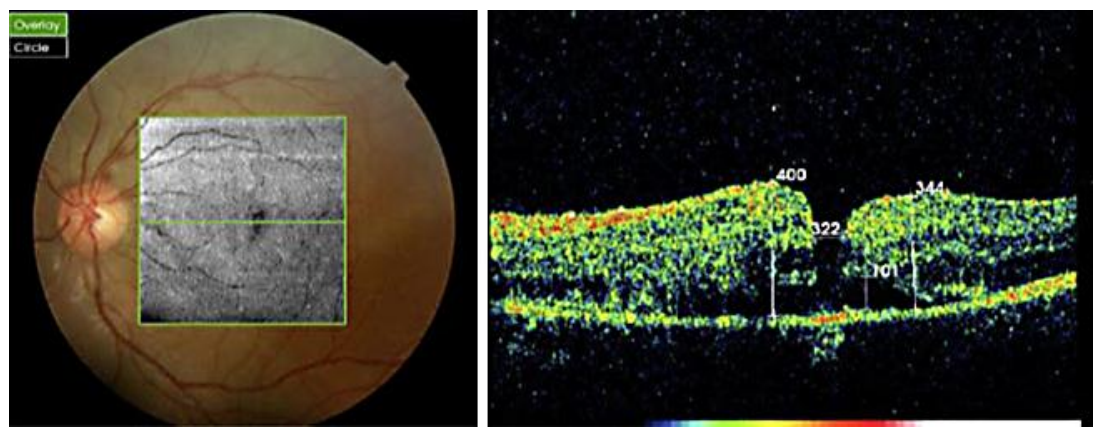

Fig. 8. Presentation: OS, full-thickness macular hole, iVA hand motion.
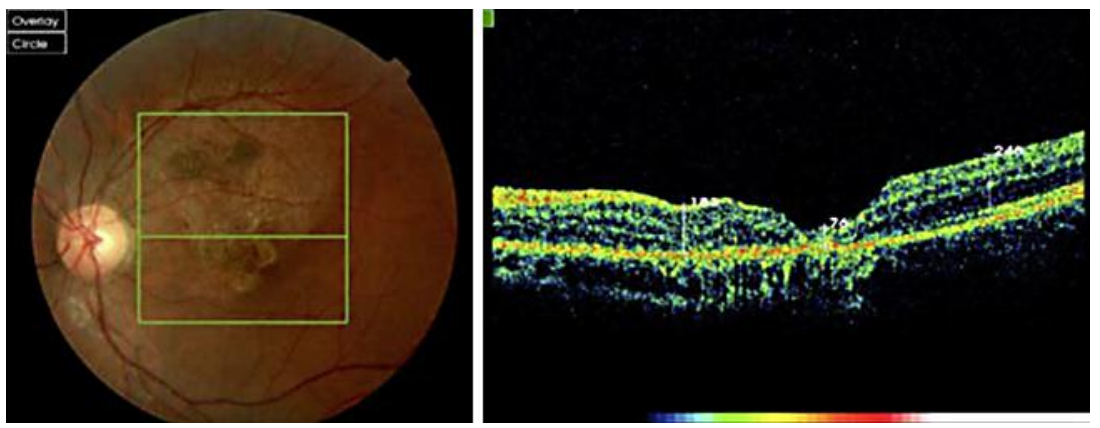

Fig. 9. One year later: macular closure, fVA counting fingers. 